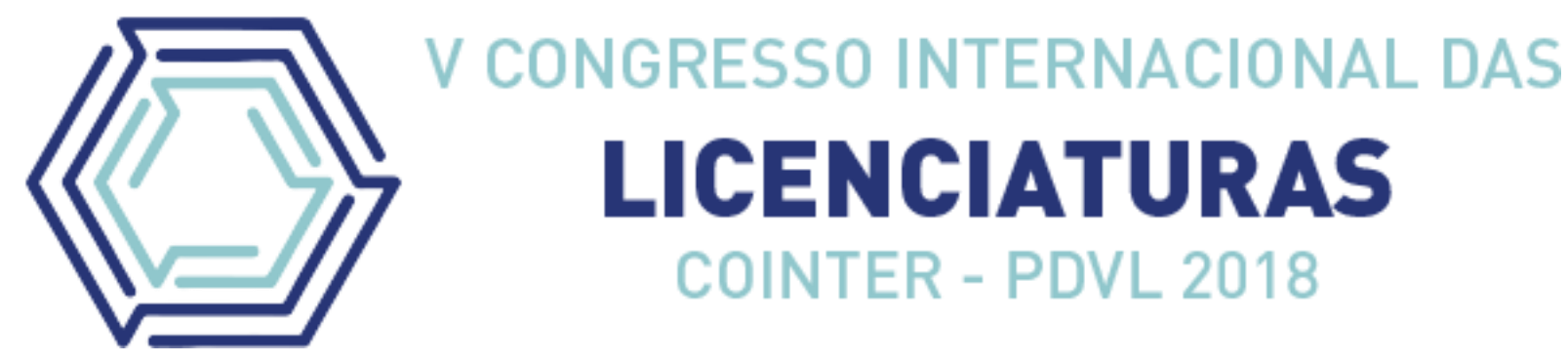

\title{
A MODELAGEM DE FENÔMENO FÍSICO POR MEIO DE FUNÇÕES POLINOMIAIS
}

\section{THE PHYSICAL PHENOMENA MODELING BY POLYNOMIAL FUNCTIONS}

\author{
Apresentação: Comunicação Oral \\ João Pedro Nogueira da Silva ${ }^{1}$; Gessika Gonçalves Ferreira ${ }^{2}$; Alinne Mayara S. de Macêdo ${ }^{3}$; \\ Laura M. dos S. M. Pereira ${ }^{4}$; Margareth Santoro B. de Oliveira ${ }^{5}$
}

\section{DOI: $\underline{\text { https://doi.org/10.31692/2358-9728.VCOINTERPDVL.2018.00016 }}$}

\section{Resumo}

Reflexões sobre a metodologia de ensino da Matemática, em todos os níveis, não são atuais e suscitam a necessidade de se repensar sobre meios que auxiliem os professores no processo ensino-aprendizagem dessa disciplina. A interdisciplinaridade e contextualização, importantes no processo formativo do licenciando em Matemática, utilizadas como estratégias conduzem, assim, a uma aprendizagem diferenciada, mais significativa e produtiva, promovendo a aproximação e a interação dos fatos da realidade com o conteúdo acadêmico. Configura-se, assim, a proposição do presente trabalho que é a investigação científica sobre o movimento de uma bola de basquete baseada na modelagem matemática a partir de funções polinomiais de graus zero, um e dois, ou seja, um diálogo entre as disciplinas de Matemática e Física numa perspectiva educacional em busca de inovação da prática docente, superando modelos tradicionais de ensino. A metodologia iniciou-se com levantamento bibliográfico a fim de ampliar a capacidade produtiva, contextualizada e criativa na posse de conceitos físicos com o interesse de trabalhar o conteúdo de funções polinomiais. O percurso foi desenvolvido a partir da descrição do movimento de uma bola de basquete por meio dessas funções, a análise e interpretação do modelo matemático. Ao final, percebeu-se que a manipulação de funções polinomiais, a partir da modelagem de um fenômeno físico, é uma estratégia de ensino que possibilita suprir necessidades do processo de aprendizagem; as ações pedagógicas podem ser pensadas de maneira que englobem o uso da modelagem matemática, como forma de uma reconstrução conceitual, ou seja, dar sentido àquilo que se deseja ensinar.

Palavras-Chave: Modelagem Matemática, Estudo do Movimento, Funções Polinomiais.

\section{Abstract}

Reflections on the teaching methodology of Mathematics at all levels are not current and raise

\footnotetext{
${ }^{1}$ Estudante de Licenciatura em Matemática, IFRN, joaopedronogueira725@gmail.com

2 Estudante de Licenciatura em Matemática, IFRN, gessikagon@outlook.com

${ }^{3}$ Estudante de Licenciatura em Matemática, IFRN, alinnemayara2009@hotmail.com

${ }^{4}$ Estudante de Licenciatura em Matemática, IFRN, laura.medeiros@academico.ifrn.edu.br

${ }^{5}$ Prof. ${ }^{\text {a }}$ MSc. em Física, IFRN/ CNAT e EPCAR, magdeoliveira@gmail.com
} 
the need to rethink about ways that assist teachers in the teaching-learning process of this discipline. The interdisciplinarity and contextualization, important in the formative process of the student of degree in Mathematics, used as strategies lead, therefore, a differentiated learning, more significant and productive, promoting the approximation and the interaction of the facts of the reality with the academic content. Thus, the proposition of the present work is the scientific investigation about the movement of a basketball based on mathematical modeling from polynomial functions of degrees zero, one and two, that is, a dialogue between the disciplines of Mathematics and Physics in an educational perspective in search of innovation of teaching practice, surpassing traditional models of teaching. The methodology began with a bibliographical survey in order to expand the productive capacity, contextualized and creative in the possession of physical concepts with the interest of working the content of polynomial functions. The course was developed from the description of the movement of a basketball by means of these functions, the analysis and interpretation of the mathematical model. In the end, it was noticed that the manipulation of polynomial functions, from the modeling of a physical phenomenon, is a teaching strategy that allows to supply needs of the learning process; pedagogical actions can be thought in a way that encompasses the use of mathematical modeling as a way of conceptual reconstruction, that is, giving meaning to what one wishes to teach.

Keywords: Mathematical Modeling, Motion Study, Polynomial Functions.

\section{Introdução}

Reflexões sobre a construção do conhecimento matemático na sala de aula suscitam a necessidade de se buscar mecanismos que auxiliem o professor no ensino-aprendizagem da Matemática. Nesse sentido, faz-se importante a contextualização e a interdisciplinaridade no processo formativo do licenciando em Matemática para que possa tornar o processo educativo da disciplina mais prazeroso, visto que este passa a ter relação com a vida cotidiana do educando ou com seus conhecimentos adquiridos anteriormente. Fernandes (s/d) enfatiza que no ensino da Matemática, a contextualização torna-se um instrumento útil quando abordada de uma forma ampla e não de modo artificial e restritivo apenas ao cotidiano do aluno.

Contudo, um aspecto que deve ser considerado é a necessidade de superação de alguns hábitos intelectuais estabelecidos por muitas práticas escolares, de concepções fragmentadas. É fundamental o abandono de uma prática mecanicista, na qual o aluno aprende técnicas aritméticas e depois realiza alguns cálculos nos quais utilizam equações não constatando sua relação com algo prático. Por isso, muitas vezes a Matemática é considerada a responsável pelo baixo desempenho escolar devido à fragilidade dos conhecimentos matemáticos por parte dos alunos. Não obstante, a questão reside na forma como a Matemática é ensinada no contexto físico (PIETROCOLA, 2002). Assim, a proposição do uso da contextualização assemelha-se ao que é realizado na Modelagem Matemática. Segundo Bassanezi (2004), a Modelagem Matemática é um processo que visa obter e validar modelos ${ }^{6}$; consiste em descrever situações da realidade em problemas matemáticos, fazendo com que os alunos possam desenvolver sua capacidade de reflexão de uma forma ampla, globalizada. Para Nascimento e Santos (2014), o entender a Matemática auxilia o sujeito na compreensão do mundo que o cerca, em situações cotidianas e em situações mais complexas.

\footnotetext{
6 Segundo Roque e Videira (2013, p.281), o "modelo matemático" de um fenômeno físico é dado por uma equação que descreve e permite prever os estados atuais e futuros dos fenômenos considerados.
} 
Nesse contexto, a proposta deste trabalho consiste em apresentar uma situação real que possa ser compreendida e/ou resolvida utilizando funções polinomiais de forma que os estudantes atribuam significações ao seu uso e contextualização. Com essa proposta pretendemos conduzir a uma aprendizagem diferenciada, mais significativa e produtiva, promovendo a aproximação e a interação dos fatos da realidade com o conteúdo acadêmico.

\section{Fundamentação Teórica}

É recorrente a reclamação de estudantes da educação básica - e até mesmo de pessoas já egressas desse nível de ensino - relacionada à utilidade da Matemática. Assuntos ditos difíceis pelos estudantes os levam a indagar qual a utilidade para sua vida e seu cotidiano, apesar dela estar presente em quase todas as situações do dia a dia, o que passa despercebido muitas vezes pelos discentes.

A Matemática possui uma linguagem própria capaz de traduzir numericamente eventos da natureza de tal forma que possam ser interpretados e analisados, fornecendo também recursos para pesquisa de fenômenos futuros. A Ciência vale-se dessa linguagem como forma de expressar seu pensamento e o formalismo matemático tem se mostrado pertinente para o propósito da elaboração de teorias em gerais, inclusive as físicas (BATISTA e MOZOLEVSKI, 2010).

Sabemos que a Matemática está estreitamente ligada à Física, Engenharia, Astronomia, Química, dentre outros, mas só foi reconhecida "no início do séc. XIX como uma ciência natural que envolve muitas aplicações e atividades de modelagem" (BELTRÃO, 2012, p. 3). A Matemática começa a ser aplicada em outras disciplinas já no $9^{\circ}$ ano do Ensino Fundamental, quando os conteúdos de Física e Química são inseridos na grade curricular. No ingresso ao Ensino Médio, é comum o estudante estranhar certos procedimentos adotados pelo professor, como afirmam Batista e Mozolevski (2010), pois muitos dos fenômenos físicos só podem ser compreendidos através da linguagem matemática e esta, muitas vezes, não é transmitida com clareza, permanecendo a prática mecanicista na qual "as atividades escolares acabam por se restringir às aplicações de formalismos matemáticos e aos exercícios numéricos extraídos das teorias" (PIETROCOLA, 2002, p. 92).

Sousa e Fávero (2003, p. 19), em uma pesquisa realizada com professores de Física do Ensino Médio da rede pública e privada do Distrito Federal, constataram que seus alunos "não veem a resolução de problemas como inerente à aprendizagem da Física, como parte do conhecimento físico e como atividade facilitadora da aprendizagem de conceitos físicos". E reconheceram que

\footnotetext{
O aluno não toma consciência de que a relação entre teoria e prática é interativa, de modo que é normal ter dificuldades em resolução de problemas, pois esta é também responsável pela aprendizagem da teoria. Ante esta falta de conscientização, o aluno recorre ao uso mecânico de fórmulas como se estivesse aí a aplicação da teoria que ele não consegue assimilar. (p. 20)
}

Este cenário também é vivenciado por parte dos alunos de graduação que, muitas vezes, não consegue contextualizar de forma conveniente conceitos matemáticos que lhes são apontados no decorrer de sua formação acadêmica. Uma argumentação para esse contexto, segundo SILVA et al. (2014), é que os professores formadores, muitas vezes, apresentam questões desconectadas da realidade e, consequentemente, os licenciandos, em sua formação, não são preparados para a realização de tarefas interdisciplinares e contextualizadas.

Segundo Bassanezi (1999, p. 9): 
$\mathrm{Na}$ verdade, a produção matemática tem ocorrido de modo supostamente desvinculado de um contexto sócio-cultural-político e com pouca preocupação em tornar-se utilitária ou mais bem definida em suas metas - o que, de certo modo, diferencia a Matemática de outras Ciências. De fato, tal produção apresenta-se como fruto exclusivo da mente humana, resultando numa linguagem que almeja essencialmente elegância e rigor.

A dificuldade na interpretação da linguagem matemática e a desvinculação de sua produção da realidade, impossibilita a capacidade criativa e questionadora sejam dos licenciandos, sejam de seus futuros alunos. Com o objetivo de melhorar a assimilação dos conteúdos que são ensinados e, como consequência, fazendo do aluno um sujeito ativo no processo de aprendizagem, surge, por volta da década de 1970 no Brasil, a Modelagem Matemática, como estratégia de ensino (FERREIRA, SILVEIRA e DA SILVA, 2013). Ela é uma ferramenta de grande utilidade para a resolução de uma situação-problema, visto que fornece os meios necessários para chegar a uma dada solução, apoderando-se da interligação da Matemática com outras áreas do conhecimento, trazendo as situações do mundo real para um contexto matemático, onde possam ser manipuladas e se ter uma certa previsão dos seus resultados.

Surge, assim, nosso interesse em contextualizar funções polinomiais - em particular as de grau um e dois - por meio da modelagem matemática de um fenômeno físico. Segundo Dante (2011), o conceito de função é relevante na Matemática e ocupa lugar de destaque em diversos ramos tais como na descrição de fenômenos físicos, biológicos, sociais, entre outros. Tais funções são ferramentas de grande importância no estudo da Cinemática, pois estão presentes principalmente em relações espaço-tempo e velocidade-tempo (NUSSENZVEIG, 2014).

Nesse trabalho empregaremos as funções polinomiais da forma das equações (I), (II) e (III), relacionando as grandezas físicas posição, velocidade e aceleração de um corpo, num dado referencial, para cada instante $t$.

$$
\begin{gathered}
f(t)=k \operatorname{com} k \in \mathbb{R}, \\
f(t)=a t+b \operatorname{com} a, b \in \mathbb{R}, \\
\mathrm{e} \\
f(t)=a t^{2}+b t+c \operatorname{com} a, b, c \in \mathbb{R}
\end{gathered}
$$

A partir da investigação dos resultados numéricos dessas relações, poderemos fazer revisões e interpretar o fenômeno que está sendo estudado.

\section{Metodologia}

Nosso estudo pautou-se na investigação científica sobre o movimento de uma bola de basquete arremessada com o propósito de abordagem do conteúdo matemático funções polinomiais de graus zero, um e dois.

Iniciamos nosso estudo com levantamento bibliográfico selecionando informações sobre o que já se tem produzido no tocante à interdisciplinaridade e contextualização tomando posse de conceitos físicos para contextualização de conteúdos matemáticos - e à modelagem matemática como estratégia de ensino, buscando aporte teórico para investigação do objeto de estudo, pois permite-nos um contato direto com aquilo que já foi escrito a respeito do que se quer estudar (MARQUES DA SILVA, 2015).

Dentre as obras consultadas, podemos destacar os trabalhos de Batista e Mozolevski (2010) e Bassanezi $(1999 ; 2002)$ que versam sobre a modelagem matemática, instrumento 
pelo qual abordamos nosso estudo.

Utilizamos como ferramenta de diálogo entre a Matemática e a Física, as funções polinomiais de primeiro e segundo graus, discutidas no trabalho de Dante (2011), descrevendo o movimento de uma bola de basquete do arremesso até atingir a cesta e, a partir daí, extraindo as informações desejadas do ponto de vista físico e matemático.

\section{Resultados e Discussão}

A situação contextualizada para modelagem foi a de analisar o comportamento de uma bola de basquete arremessada, com velocidade inicial de módulo $v_{0}$, sendo $\theta$ o ângulo entre $\vec{v}_{0}$ e a direção $O x$ (Figura 1). Considerando a aceleração gravitacional, próximo à superfície terrestre, de módulo constante e igual a $g$, e desprezando a resistência do ar, descrevemos matematicamente a posição da bola para cada instante $t$ e a dependência temporal de sua altura em relação ao seu deslocamento horizontal, adotando-se o sistema de coordenadas descrito na Figura 1.

Figura 1: Arremesso de uma bola de basquete. Fonte: https://brainly.com.br/tarefa/13240516.

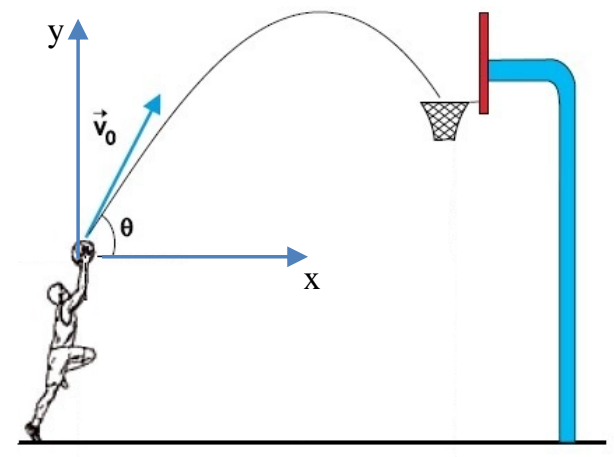

Considerando desprezível a resistência do ar, a única força que atua sobre a bola, em seu deslocamento até a cesta, é a força peso, cuja direção é vertical e sentido de cima para baixo. Pela segunda lei de Newton, temos

$$
\vec{R}=m \cdot \vec{a}
$$

em que $\vec{R}$ é o vetor resultante das forças que atuam no corpo de massa $m$ e $\vec{a}$, seu vetor aceleração. Logo,

$$
\begin{aligned}
\vec{P} & =m \cdot \vec{a} \\
\vec{a} & =-g \hat{\jmath}
\end{aligned}
$$

Pela Figura 1, o vetor velocidade inicial $\vec{v}_{0}$ pode ser escrito como:

$$
\vec{v}_{0}=\left(v_{0} \cos \theta\right) \hat{\imath}+\left(v_{0} \operatorname{sen} \theta\right) \hat{\jmath}
$$

Sabendo que a grandeza física aceleração mede a taxa de variação da velocidade da bola em relação ao tempo e $\vec{a}=-g \hat{\jmath}$, cujo módulo é constante, ou seja, independe da variável $t$ - função polinomial de grau zero. Temos:

$$
\frac{d \vec{v}}{d t}=\vec{a}
$$


Integrando obtemos $\vec{v}(t)=-g t \hat{\jmath}+c$, em que $c$ é uma constante de integração, cujo valor corresponde a velocidade inicial, para $t=0$. Logo, o vetor velocidade instantânea da bola pode ser descrito por

$$
\vec{v}(t)=\left(v_{0} \cos \theta\right) \hat{\imath}+\left(v_{0} \operatorname{sen} \theta-g t\right) \hat{\jmath}
$$

O módulo $\left(v_{0} \cos \theta\right)$ de sua componente horizontal em (IX) é uma função constante (isto é, independente do tempo $t$ ) - função polinomial de grau zero - e o módulo $\left(v_{0} \operatorname{sen} \theta-g t\right)$ de sua componente vertical em (IX), uma função polinomial do primeiro grau. A velocidade instantânea é a grandeza física que mede a taxa temporal da variação da posição da bola, em relação ao solo, ou seja,

$$
\vec{v}(t)=\frac{d \vec{s}}{d t}
$$

Logo, integrando e sendo a constante de integração a posição inicial na origem do sistema de referência para $t=0$, podemos escrever o vetor posição:

$$
\vec{s}(t)=\left(v_{0} \cos \theta t\right) \hat{\imath}+\left(v_{0} \operatorname{sen} \theta t-g \frac{t^{2}}{2}\right) \hat{\jmath}
$$

A função descrita em (XI) fornece a posição da bola, tanto em relação a vertical quanto a horizontal, para cada instante $t$. O módulo das componentes vertical e horizontal do vetor posição são, respectivamente

$$
\begin{gathered}
y(t)=v_{0} \operatorname{sen} \theta t-g \frac{t^{2}}{2} \\
\mathrm{e} \\
x(t)=v_{0} \cos \theta t
\end{gathered}
$$

e fornecem a altura e a distância percorrida na horizontal para um instante $t$.

Isolando o valor de $t$ em (XIII) e substituindo esse valor encontrado na equação (XII) temos:

$$
t=\frac{x}{v_{0} \cos \theta} \Rightarrow y(t)=v_{0} \operatorname{sen} \theta \cdot \frac{x}{v_{0} \cos \theta}-g \frac{\left(\frac{x}{v_{0} \cos \theta}\right)^{2}}{2}
$$

Logo, podemos escrever:

$$
y(t)=\operatorname{tg} \theta x-g \frac{x^{2}}{2 v_{0}^{2} \cos ^{2} \theta}
$$

Essa função é uma função polinomial de grau dois que relaciona a altura da bola, em relação ao solo, com seu deslocamento na horizontal, para um instante $t$ qualquer. 
A investigação da equação (XIV) fornece uma aproximação expressiva da trajetória da bola, desde o ponto do arremesso até atingir a cesta. Por meio da análise matemática do gráfico da função polinomial de grau dois, reconhecemos que essa trajetória é um arco de parábola com concavidade voltada para baixo. Esse resultado matemático tem a interpretação física de que o vetor aceleração da bola tem módulo constante (pois a concavidade não se altera para qualquer instante $t$ ), além de podermos determinar vários outros parâmetros físicos como o tempo que a bola permanece no ar (relaciona-se com os zeros da função), a altura máxima atingida por ela (nesse ponto, a componente da velocidade na direção vertical é nula e corresponde a ordenada do vértice da parábola - ordenada do ponto de máximo, pois a parábola tem concavidade voltada para baixo), qual a posição que o jogador deve ocupar para pontuar, entre outros. Os zeros das funções, seus pontos de mínimo e máximo (quando houver), a concavidade da curva gráfica (para a função polinomial de grau dois), representam resultados matemáticos com interpretação física.

O movimento da bola pode ser interpretado recorrendo a equação (IX). A componente do vetor velocidade instantânea, na direção horizontal, independe do tempo; logo, o movimento descrito pela bola é classificado como uniforme e o gráfico corresponde a de uma função polinomial de grau zero, ou seja, uma função constante. Sua componente vertical é dependente do tempo $t$; o movimento descrito pela bola é uniformemente variado e o gráfico é o de uma função polinomial de grau um, isto é, uma reta formando um determinado ângulo com o eixo horizontal. O movimento ocorre em duas dimensões e a bola executa, simultaneamente, dois movimentos: uniforme, na direção horizontal, e uniformemente variado, na vertical (composição de movimentos).

\section{Conclusões}

A motivação deste trabalho veio da oportunidade de integrar conhecimentos das disciplinas de Matemática e Física por meio da contextualização e problematização. A proposição é de fornecer subsídios para o licenciando, futuro professor de Matemática, de forma a expressar a possibilidade de exposição de conceitos matemáticos a partir da descrição de fenômenos físicos com a finalidade de corrigir a fragmentação das disciplinas, por meio da interdisciplinaridade, propiciando aos seus futuros alunos, à vista disso, uma postura crítica e um aprendizado significativo em sua formação.

Baseado numa situação real física, exemplificada pela análise do movimento de uma bola de basquete ao ser arremessada para cesta, visou-se desenvolver o pensamento crítico e investigador do discente, pois permitiu-nos descrever e prever os estados atuais e futuros do fenômeno considerado. Assim, utilizando equações paramétricas na variável tempo $t$ (funções polinomiais), obtivemos a equação da trajetória descrita por uma bola, ou por um projétil, em movimento livre não vertical, em um campo gravitacional uniforme, e reconhecemos que essa trajetória é, de fato, um arco de parábola com concavidade voltada para baixo.

A manipulação de funções polinomiais, a partir da modelagem de um fenômeno físico, é uma estratégia de ensino que possibilita suprir necessidades do processo de aprendizagem; as ações pedagógicas podem ser pensadas de maneira que englobem o uso da modelagem matemática, como forma de uma reconstrução conceitual, ou seja, dar sentido àquilo que se deseja ensinar.

O propósito não é somente fazer com que o conteúdo matemático seja assimilado pelo discente, mas de situar os licenciandos em seu papel de mediador junto ao aluno, no processo de apropriação do conhecimento. Deste modo, a Matemática é inclusa no contexto social, 
tornando-se interessante, eficiente e sedutora, tanto para os futuros professores como para os alunos, ampliando assim as possibilidades de compreensão e transformação da realidade.

\section{Referências}

BASSANEZI, Carlos Rodney. Ensino-aprendizagem com modelagem matemática: uma nova estratégia. 2. ed. - São Paulo: Contexto, 2004.

BASSANEZI, R. C. Modelagem matemática: Uma disciplina emergente nos programas de formação de professores. Biomatemática IX (1999) p. 9-22. Disponível em: <http://www.somaticaeducar.com.br/arquivo/material/142008-11-01-15-57-39.pdf >. Acesso em: 19 de setembro 2018.

BATISTA, Eliezer; MOZOLEVSKI, Igor. Métodos de Física-Matemática. Florianópolis: Universidade Federal de Santa Catarina, 2010.

BELTRÃO, Maria Eli Puga. Aplicações e modelagem matemática: aspectos históricos. In: SEMINÁRIO INTERNACIONAL DE PESQUISA EM EDUCAÇÃO MATEMÁTICA, 5, Petrópolis, 2012. Anais. Sociedade Brasileira de Educação Matemática, 2012.

DANTE, Luiz Roberto. Matemática: contexto e aplicações. ed. 1, v. 1, São Paulo: Ática, 2011, 504 p.

FERNANDES, Suzana da Silva. A contextualização no ensino de matemática - um estudo com alunos e professores do ensino fundamental da rede particular do distrito federal. (s/d). Disponível em: <http://www.ucb.br/sites/100/103/TCC/22006/SusanadaSilvaFernandes.pdf〉. Acesso em: set. 2018

FERREIRA, G. P., SILVEIRA, A., DA SILVA, L. A. A modelagem matemática ao longo da história e o surgimento da modelação matemática no Brasil. Anais do XI Encontro Nacional de Educação Matemática - ISSN 2178-034X Encontro Nacional de Educação Matemática. Educação Matemática: Retrospectivas e perspectivas. Julho de 2013. Curitiba-PR

LOPES, C. E. Os Desafios e as Perspectivas para a Educação Matemática no Ensino Médio. Trabalho encomendado pelo GT19 - Educação Matemática. Apresentação da 34a Reunião Anual da ANPED. [s.1.], 2011.

MARQUES DA SILVA, Airton. Metodologia da Pesquisa. 2 ed, Fortaleza: EdUECE, 2015. $107 \mathrm{p}$.

NASCIMENTO, Diná Ester Barbosa do; SANTOS, Maria José Costa dos. Matemática na EJA através da metodologia da sequência de Fedathi e do livro paradidático. In: FÓRUM INTERNACIONAL DE PEDAGOGIA, 6, Santa Maria, 2014. Anais. Associação Internacional de Pesquisa na Graduação em Pedagogia, 2014, n.p.

NUSSENZVEIG, Herch Moysés. Curso de Física Básica, 2: fluidos, oscilações e ondas, calor. 5. ed. SP: Blucher, 2014. 
PIETROCOLA, M. A Matemática como Estruturante do Conhecimento Físico. Caderno Catarinense de Ensino de Física, v. 19, n.1: p.89-109, ago. 2002.

ROQUE, T., VIDEIRA, A. A. P. A Noção de Modelo na Virada do século XIX para o século XX Sci. Stud. [online], v. 11, n. 2, p.281-304, 2013.

SILVA, J. R. da, et al. As idealizações dos cálculos de Newton e Leibniz como organizadores prévios comparativos para a definição de derivada. Aprendizagem significativa em Revista/Meaningful Learning Review -v.4, n.2, pp. 57-71, 2014.

SOUSA, C. M. S. G., FÁVERO, M. H. Concepções de Professores de Física sobre Resoluções de Problemas e o Ensino de Física. Revista Brasileira de Pesquisa em Educação em Ciências, v. 3, n. 1, p. 58-69, 2003. 